\title{
Treatment of Congenital Idiopathic Clubfoot: Ponseti Method
}

\author{
Dr. Md. Abdus Sobhan ${ }^{1 *}$, Dr. Md. Zulfiqur Ali², Dr. Nahid Sultana ${ }^{3}$
}

${ }^{1}$ Senior Consultant, Ortho Surgery (Regular), Adhunik Sadar Hoapital Chapinawabgonj, Bangladesh

${ }^{2}$ Assistant Professor, Department of Orthopedics, Shaheed M. Monsur Ali Medical College, Sirajganj, Bangladesh

${ }^{3}$ Assistant Professor, Department of Gynae \& Obstetrics, Rajshahi Medical College, Rajshahi, Bangladesh

DOI: $10.36347 /$ sjams.2020.v08i06.035

| Received: 20.06.2020 | Accepted: 27.06.2020 | Published: 30.06.2020

*Corresponding author: Dr. Md. Abdus Sobhan

Abstract

Clubfoot is a birth defect that is marked primarily by a deformed talus (ie, ankle) and calcaneous (ie, heel) that give the foot a characteristic "club-like" appearance. In congenital idiopathic clubfoot (ie, talipes equinovarus), the infant's foot points downward (ie, equinus) and turns inward (ie, varus), while the forefoot curls toward the heel (ie, adduction). This congenital disorder has an incidence of 1 in 400 live births, with boys affected twice as often as girls. Unilateral clubfoot is somewhat more common than bilateral clubfoot and may occur as an isolated defect or in association with other disorders (eg, chromosomal aberrations, cerebral palsy, spina bifida, arthrogryposis). Our study aim was to evaluate Ponseti Method for The Management of Congenital idiopathic clubfoot. This was a descriptive crosssectional study conducted in the dept. of orthopaedics, Adhunik Sadar hospital chapainawabganj, Bangladesh during the period from January 2017 to June 2018. Data was collected from patients with idiopathic clubfoot, presenting at selected hospital. Both, unilateral, bilateral were included in the study. In this this study, we have treated 50 children with idiopathic clubfoot by Ponseti method of correction of serial manipulation and casting. Among the 50 children, 24 had unilateral and 22 bilateral involvement. Male were 26 and remaining 20 were female. Most children had grade III and grade IV (Dimeglio et al., grading) deformity. The mean number of casts required was 5.76 (4 to 10). Tenotomy was required in 12 feet (40\%). The scores for the entire group ranged from 4.0 to 7.0 (of 10) and 4 to 19 (of 20) in the Dimeglio classification. Pirani scores for the age group 0 - 6 months were 4.46, 6 months to 1 year were 5.36 and 1-2 years were 5.75 respectively. The results were excellent in $88.3 \%$ and good in $11.7 \%$. Initial and final scores were statistically evaluated with paired ' $\mathrm{T}$ ' test with a ' $\mathrm{T}$ ' value of 36.76 with a ' $\mathrm{P}$ ' value of less than 0.001 . Thus there was a significant reduction in the score from the pre level because of intervention. Results: Similar good results and low requirement for surgical interventions other than Achilles tenotomy, which forms part of the Ponseti regimen, were found. In terms of number of patients the success rate is $87 \%$ and in terms of the number of feet the success rate is $91 \%$. This study demonstrates that treatment of congenital talipes equine varus by conservative management of ponseti method can be sufficient.

Keywords: Clubfoot, Ponseti Method, Foot Deformities, Congenital.

Copyright @ 2020: This is an open-access article distributed under the terms of the Creative Commons Attribution license which permits unrestricted use, distribution, and reproduction in any medium for non-commercial use (NonCommercial, or CC-BY-NC) provided the original author and source are credited.

\section{INTRODUCTION}

Clubfoot refers to a condition in which a newborn's foot or feet appear to be rotated internally at the ankle. The foot points down and in wards, and the soles of the feet face each other. It is known as talipes equinovarus (TEV) or congenital talipes equinovarus (CTEV). In 50 percent of cases, both feet are affected. Clubfoot is a birth defect that is marked primarily by a deformed talus (ie, ankle) and calcaneous (ie, heel) that give the foot a characteristic "club-like" appearance. In congenital idiopathic clubfoot (ie, talipes equinovarus), the infant's foot points downward (ie, equinus) and turns inward (ie, varus), while the forefoot curls toward the heel (ie, adduction). This congenital disorder has an incidence of 1 in 400 live births, with boys affected twice as often as girls Club foot, or talipes equinovarus, is a congenital deformity consisting of cavus, forefoot adduction, heel varus, ankle equinus and internal tibial torsion. The deformity was described as early as the time of Hippocrates. The term talipes is derived from latin talus-ankle, and pes-foot [1]. The term refers to the gait of severely affected patients, who walked on their ankles. The goal of treatment is to correct all components of the deformity so that the patient has a pain-free plantigrade foot with good mobility, without calluses, and without the need to wear special or modified shoes. In 1889, L.H. Bradford has been quoted as saying. "The literature on the treatment of clubfoot is, as a general rule, that of unvarying success. It is often as brilliant as an advertising sheet and yet in 
practice there is no lack of half-cured or relapses cases, sufficient evidence that method of cure are not universally understood. "More than a hundred years on, these words, unfortunately, still ring true.

\section{Details of Ponseti Technique [2]}

Treatment is ideally started soon after birth by passive manipulations which are taught to the parents till casting is started after $7-10$ days.

1. First cast (reduce the cavus): The first element of management is correction of the cavus deformity by positioning the forefoot in proper alignment with the hindfoot.

2. Manipulation: The manipulation consists of abduction of the foot beneath the stabilized talar head. Locate the head of the talus. All components of clubfoot deformity, except for the ankle equinus, are corrected simultaneously. To gain this correction, one must locate the head of the talus, which is the fulcrum for correction.

3. Second, Third and Fourth Casts: During this phase of treatment, the adductus and varus are corrected. The distance between the medial malleolus and the tuberosity of the navicular when palpated with the fingers tells the degree of correction of the navicular. When the clubfoot is corrected, that distance measures approximately 1.5 to $2 \mathrm{~cm}$ and the navicular covers the anterior surface of the head of the talus. Similarly, the extent of the lateral displacement of the anterior tuberosity of the calcaneus under the head of the talus indicates the increase in value of the talo-calcaneal angle, and thus, the degree of correction of the heel varus.

\section{Equinus}

The equinus deformity gradually improves with correction of adductus and varus. This is part of correction because the calcaneus dorsiflexes as it abducts under the talus. No direct attempt at the equinus correction is made until the heel varus is corrected. In very flexible feet, equinus may be corrected by additional casting without tenotomy when in doubt, perform the tenotomy.

\section{Cast Application, Moulding and Removal}

Success in ponseti management requires good casting technique. Those with previous club foot casting experience may find it easier than those learning clubfoot casting for the first time.

\section{End-Point}

The endpoint was a score less than 1.5 with the feet having 70 degrees of external rotation and 15 degree of dorsiflexion. The parents were advised to preserve the plaster casts as they served as replica for assessing the correction.

\section{Bracing Protocol}

The brace is applied immediately after the last cast is removed, 3 weeks after tenotomy. The brace consists of open toe high-top straight last shoes attached to a bar. For unilateral cases, the brace is set at 60 to 70 degrees of external rotation on the clubfoot side and 30 to 40 degrees on the normal side. On bilateral cases, it is set at 70 degrees of external rotation on each side. The bar should be of sufficient length so that heels of the shoes are at shoulder width. A narrow brace is a common reason for lack of compliance. The bar should be bent 5 to 15 degrees with the convexity away from the child to hold the feet in dorsiflexion. The brace should be worn full time (day and night) for the first 3 months after the last cast is removed. After that, the child should wear the brace for $12 \mathrm{hrs}$ at night and 2 to $4 \mathrm{hrs}$ in the middle of the day for a total of 14 to $16 \mathrm{hrs}$ during each 24 -hr period. This protocol continues until the child is 3 to 4 years of age.

\section{OBJECTIVES \\ General Objective}

- To evaluate The Treatment of Congenital idiopathic clubfoot by Ponseti Method

\section{Specific Objective:}

- To know more about clubfoot scenarios in Bangladesh

- To know the treatment options of Congenital idiopathic clubfoot

\section{Materials And Methods}

This was a descriptive cross-sectional study in the dept of orthopaedics, Adhunik Sadar hospital chapainawabganj, Bangladesh during the period from January 2017 to June 2018. We selected 50 participants for this study. Our aim was to evaluate The Treatment of Congenital idiopathic clubfoot Ponseti Method and tried to find out better treatment options for congenital idiopathic clubfoot problem.

\section{Pirani Severity Scoring}

We have used the pirani severity scoring3 developed by Catterall and Shafique pirani to evaluate the correction in an unoperated congenital clubfoot less than 2 year of age. This rating has proved its efficacy in having little inter and intra-observer variability. Documenting the amount of deformity allows the treating practitioner where he or she is with respect to the road map of treatment, to know when tenotomy is indicated, and to reassure parents regarding progress. It allows meaningful comparison of results, extractions of subgroups, etc.

\begin{tabular}{|l|l|}
\hline Scores & Clinical Signs \\
\hline 0 & Normal \\
\hline 0.5 & Moderately normal \\
\hline 1 & Severely abnormal \\
\hline
\end{tabular}

Midfoot Score. Three signs comprise the midfoot score (MS), grading the amount of midfoot deformity between 0 and 3 
1. Curved lateral border $[\mathrm{A}]$

2. Medial crease [B]

3. Talar head coverage[C]

Hindfoot score. Three signs comprise the Hindfoot score (HS), grading the amount of Hindfoot deformity between 0 and 3

1. Posterior crease [D]

2. Rigid equines [E]

3. Empty heel $[\mathrm{F}]$

Follow-Up

After applying the brace for the first time when the last cast is removed the child returns according to the following suggested schedule

1. Weeks to troubleshoot compliance issues

2. Months to graduate to the nights- and - naps protocol

3. Every 4 months until age 3years to monitor compliance and check for relapses

4. Every 6 months until age 4 years

5. Every 1 to 2 years until skeletal maturity

Once the child is above one year of age, he is put on clubfoot shoes. These characteristically are an ankle foot orthoses, with a straight medial border, lateral wedge, no heel and an ankle strap with a gap at the point of heel to monitor the correct dorsiflexed position of the ankle through the shoes. The Dennis brown splint is still worn at night for four hours. Whenever possible slight manipulations are done by parents.

\section{RESULTS}

The initial pirani score ranged from 5-6. The number of casts ranged from 6-8. All the patients had above-knee casts. Throughout the course of plaster treatment special care was taken to counsel the parents regarding their role in watching for vascular and other plaster related complications. Clubfoot correction was obtained in all patients. Fortunately no patient had too severe a deformity that evaded correction. The endpoint of correction was defined to be achieved if the feet had a score less than 1.5 with full correction of equinus. Number of Casts Correction was obtained with 6-8 casts. $63 \%$ feet required 6 casts for correction. The last cast was usually left on for 2 weeks if a tendoachilles tenotomy was done. $13 \%$ feet required 8 casts for correction and this was indicated because of minor complications and the severity of the deformity. This corroborates with the world literature where ponseti's method usually achieves plaster correction with 5 or 6 casts. Duration The average time from the first cast to the tendoachilles tenotomy/full correction of the deformity was 42 days. The parent compliance with the bracing protocol was remarkable. They were sounded as to their role in maintaining the correction with proper fitting shoes and changing the shoe size as the feet grew. All patients have had Percutaneous Tenotomy. We had a patient population less than 12 months of age with an average of less than 1 months and all the feet responded well to the technique protocol. Average post tenotomy ankle dorsiflexion was 15 degrees. Follow up: A minimum follow-up of 12 months was possible with all the patients (Fig-1). The majority of patients started walking at the age of 16-18 months. 19 patients were followed up for 12 months and 11 patients up to $16-18$ months within the timespan of the study. Strict concordance to the protocol for follow-up outlined by ponseti was maintained throughout the study. At each follow-up the feet were evaluated for any early sign of relapse.

\section{Failure}

Failure Two patients had an average final score of 2. The reason for failure in those patients could be attributed to defective casting technique.

\section{Complications}

21 patients out of 50 had minor complications. All the complications were due to defective plastering technique and were unique to initial patients. Hence in this study, as there is only 3 patients out of 50 patients who had failure, ponseti's method was a success [6-8]. In terms of number of patients the success rate is $93 \%$. This is in good agreement with the values in literature.

Table-1: Background characteristics of the study participants $(n=50)$

\begin{tabular}{|l|l|l|}
\hline Characteristics & $\mathbf{n}$ & $\%$ \\
\hline Sex & 28 & 56.52 \\
\hline Male & 22 & 43.48 \\
\hline Female & \multicolumn{1}{|l|}{} \\
\hline All patients were younger than 12 months of age \\
\hline The number of casts range & 6 & 8 \\
\hline Involvement & 26 & 52.17 \\
\hline Unilateral & 24 & 47.83 \\
\hline Bilateral &
\end{tabular}




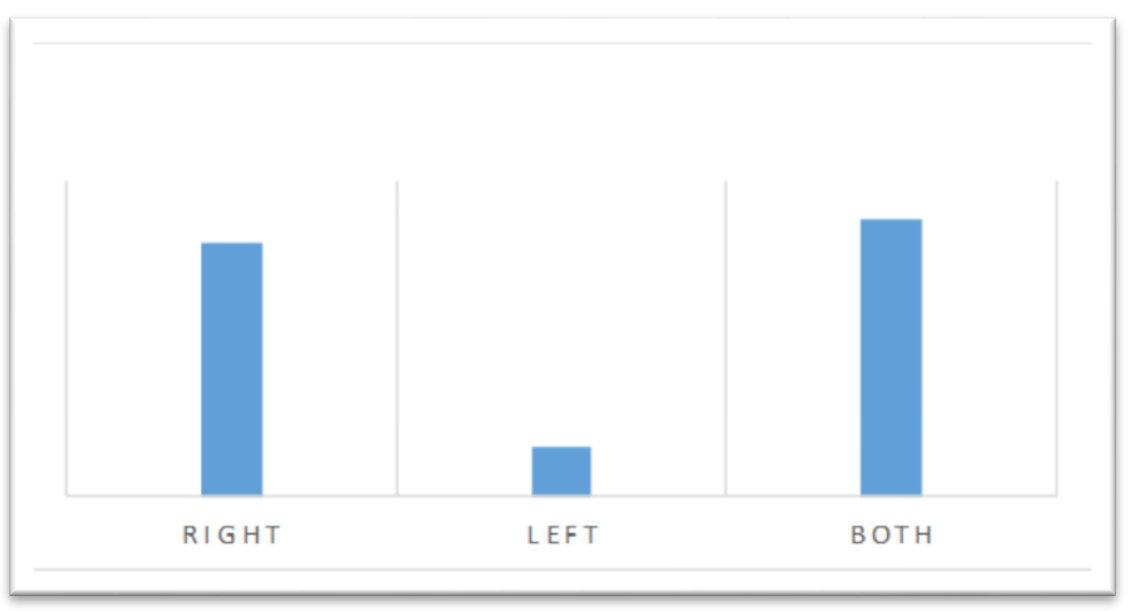

Fig-1: Side involvement of the study participants $(\mathrm{n}=\mathbf{5 0})$

Table-2: Standard Deviation

\begin{tabular}{|l|l|l|l|l|l|}
\hline & N & Mean & SD & Maximum & Minimum \\
\hline Pirani scoring_Baseline & 50 & 5.58 & .32 & 5 & 6 \\
\hline Pirani scoring- Final & 50 & 0.36 & .63 & 0 & 2.25 \\
\hline
\end{tabular}

Table-3: Cohort analysis during the study $(n=50)$

\begin{tabular}{|l|l|l|l|l|}
\hline Period & Min-Max & Mean \pm SD & Difference from starting & p value \\
\hline Starting & $5-6$ & $5.72 \pm 0.28$ & NA & NA \\
\hline 1st week & $4-5.5$ & $4.66 \pm 0.88$ & 1 & 0.001 \\
\hline 2nd week & $3-5$ & $3.53 \pm 1.28$ & 1.5 & 0.001 \\
\hline 3rd week & $2-4.5$ & $2.84 \pm 1.46$ & 2 & 0.001 \\
\hline 4th week & $1-4.1$ & $1.69 \pm 1.74$ & 2.5 & 0.001 \\
\hline 5th week & $0-3.5$ & $1.03 \pm 1.01$ & 2.5 & 0.001 \\
\hline 6th week & $0-3$ & $0.96 \pm 1.65$ & 3 & 0.001 \\
\hline 7th week & $0-2.5$ & $0.87 \pm 1.22$ & 3.5 & 0.001 \\
\hline 8th week & $0-2.5$ & $0.58 \pm 0.42$ & 3.5 & 0.001 \\
\hline Final stage & $0-2.5$ & $0.37 \pm 0.63$ & 3.5 & 0.001 \\
\hline
\end{tabular}

\section{DiscUSSION}

A conservative method of management of idiopathic CTEV has been recommended by most paediatric orthopedicians in the early days of infancy starting as early as possible after birth. There have been many conservative protocols of management for idiopathic CTEV deformity. Traditional9, Kite [10] and Ponseti [11] methods are among the commonly used protocol treatment methods. In the recent years the Ponseti method is the most advocated method of the conservative management of idiopathic CTEV deformity. Several studies have been conducted to prove its efficacy [12]. After serial manipulation and cast treatment the maintenance of correction is an important issue. Various foot abduction orthoses such as Dennis Brown splint [13], Stenbek Foot abduction orthoses and Dobb's brace have been suggested as essential orthotic management for the prevention of recurrence. We, in our study evaluated the effectiveness of Ponseti casting as a mode of correction and maintenance of idiopathic CTEV deformity. There were altogether 30 patients with 44 feet included in the study. We evaluated the following variables. Age of the patient at first visit to our institution, previous treatment and type of treatment before referral, Number of casts, Number of casts required at our institution, Need for percutaneous tendoachilles tenotomy and Compliance with the foot -abduction brace. These variables were in turn related to the need for corrective surgery and the incidence of relapses. Fisher exact tests, $t$ tests, and odds ratio were used as appropriate. The following are the outstanding bio statistical data derived from the study. A total of 26 (83 percent) patients were male, Kite in his series of 1509 cases has reported $70 \%$ males and 30\% females [14]. In Turco's [15] series of 468 patients there were $334(71.36 \%)$ males and $134(28.63 \%)$ females a sex ratio of $2.5: 1$; Male: Female. 16 patients had unilateral feet and thus unilateral feet exceeded bilateral feet in this study. Of the unilateral feet 9 were right and 7 left, Chung [16] observed a $50 \%$ bilaterality 30 Turco's series of 468 pts. Had $56 \%$ bilateral, $22 \% \mathrm{rt}$ and $21 \% \mathrm{Lt}$ side involvement. At initial ponseti casting, all patients were younger than 12 months of age. The initial ponseti score ranged from $5-6$. A total of 20 patients had some form of treatment before their initial visit to our institution. All the twenty had serial manipulation and casting done 
elsewhere. The number of casts ranged from $2-$ 10.Stephen MD has reported an average of 2.5months of treatment in his series of 37 pts. Fifty percent of these patients had below -knee casts and the rest had above-knee casts. All came to our clinic with all of the components of the deformity uncorrected. Throughout the course of plaster treatment special care was taken to counsel the parents regarding their role in watching for vascular and other plaster related complications. This counseling was effective and on several occasions the parents had contacted over phone from distant places and adequate advice could be given.

\section{Limitations of the Study}

This was a single center study with a small sample size, which may not be generalize in the whole country.

\section{CONCLUSION AND \\ RECOMMENDATIONS}

We can be concluded that Ponseti's method is a patient friendly and economically compliant method of easy and early correction of the club foot deformity. It is based on a strong scientific understanding of what the deformity is due to and what it is made up of. The results of our study closely match that available in literature which is an evidence of reproducibility of the correction irrespective of individual variations. There is a limited but important learning curve for perfecting the method which is crucial to pass through to avoid basic errors as outlined by Ponseti as committable and preventable.

\section{REFERENCES}

1. Ponseti. Ponseti IV. Treatment of congenital clubfoot. J Bone Joint Surg. 1992; 74A:448.

2. Ponseti IV. Treatment of congenital clubfoot. J Bone Joint Surg Am. 1992; 74:448-454.

3. Pirani S, Outerbridge H, Moran M. A method of evaluating the virgin clubfoot with substantial interobserver reliability. Presented at the annual meeting of the Pediatric Orthopaedic Society of North America, Miami, Florida, 1995.

4. Tarraf IN, Carrot NC, Analysis of the components of residual deformity in clubfeet presenting for reoperation. J Paediatr Orthop. 1992; 12:207-216.

5. Caroll NC, Mc Murtky R, Leete SF. The pathoanatomy of congenital clubfoot orthopedic clinics of North America. 1995; 9:2255.

6. Vesely DC. A method of application of a clubfoot cast. Clin Orthop. 1972; 84:47-49.

7. Karski T, Wogko 1. Experience in the conservative treatment of congenital clubfoot in newborns and infants. J Pediatr Orthop. 1989; 9:134-136.

8. Colburn M, Williams M. Evaluation of the treatment of idiopathic clubfoot by using the Ponseti method. J Foot Ankle Sung. 2003; 42(5):259-67.

9. Herzenberg JE, Radler C, Bor N. Ponseti versus traditional methods of casting for idiopathic clubfoot J Pediatric Orthop B. 2003; 12:133-140.

10. Kite JH. The clubfoot. London: William (Heinmann) Medical Books Ltd, 1964.

11. Dobbs MB, Morcuende JA, Gurnett CA, Ponseti IV. Treatment of idiopathic clubfoot: an historical review. Iowa Ortho pJ. 2000; 20:59-64.

12. Staheli Clubfoot L. Ponseti management 3rd ed, Seattle, Washington: Global Help Publications, 2009.

13. Hattori T, Ono Y, Kitakoji T, Iwata H. Effect of the Dennis Browne splint in conservative treatment of congenital clubfoot J Pediatric Orthop B. 2003; 12:59-62.

14. Kite JH Principles in the treatment of congenital clubfoot J. Bone Joint Surgery. 1939; 21:595-606.

15. Turco VJ. Resistant congenital clubfoot American Academy of Orthopedic Surgeons Instructional Course Lectures, St Louis the CV Mosby Co. $1975 ; 24$.

16. Chung CS, Nemechek RW, Larsen IJ, Chung GHS. Genetic and epidemiological studies of clubfoot in Hawaii Human Heredity. 1969; 19:321. 